\title{
Serum Free Triiodothyronine Is Inversely Associated With Diabetic Peripheral Neuropathy but Not With Carotid Atherosclerotic Lesions in Type 2 Diabetic Patients With Euthyroid Function
}

\author{
Mei-Fang Li \\ Shanghai Sixth Peoples Hospital \\ Jiang-Feng Ke \\ Shanghai Sixth Peoples Hospital \\ Shuai Li \\ Shanghai Sixth Peoples Hospital \\ Jun-Wei Wang \\ Shanghai Sixth Peoples Hospital \\ Zhi-Hui Zhu \\ Shanghai Sixth Peoples Hospital \\ Jing-Bo Li ( $\approx$ lijb@sjtu.edu.cn ) \\ Shanghai Sixth Peoples Hospital
}

\section{Research Article}

Keywords: free triiodothyronine, diabetic peripheral neuropathy, carotid atherosclerotic lesions, type 2 diabetes, euthyroid function

Posted Date: August 10th, 2021

DOI: https://doi.org/10.21203/rs.3.rs-773386/v1

License: (ㅇ) (1) This work is licensed under a Creative Commons Attribution 4.0 International License. Read Full License

Version of Record: A version of this preprint was published at Diabetology \& Metabolic Syndrome on December 1st, 2021. See the published version at https://doi.org/10.1186/s13098-021-00760-2. 


\section{Abstract}

Background: The associations between serum free triiodothyronine (FT3) and diabetic peripheral neuropatprohy (DPN) / carotid atherosclerotic lesions in type 2 diabetic patients with normal thyroid function is still unclear. The purpose of our study was to explore the relationships of FT3 with DPN and carotid atherosclerotic lesions in Chinese type 2 diabetes inpatients with euthyroid function.

Methods: A total of 2477 type 2 diabetes inpatients with euthyroid function were recruited in this cross-sectional study, and they were stratified into quartiles by FT3 levels. Peripheral neuropathy was assessed by neurological symptoms and signs as well as nerve conduction velocity tests. Carotid atherosclerotic lesions, including carotid intima-media thickness, plaque and stenosis, were evaluated by Doppler ultrasound.

Results: After adjusting for potential confounders, there was a significant decrease in the prevalence of DPN in the patients with type 2 diabetes across the FT3 quartiles $(23.5 \%, 20.9 \%, 18.9 \%$, and $11.2 \%$, respectively, p < 0.001). Logistical regression analysis further revealed that FT3 quartiles were significantly and inversely associated with DPN. Compared with the subjects in the highest FT3 quartile, the adjusted odds ratios $(95 \%$ confidence interval) of DPN in the first to third FT3 quartile were successively 2.338 (1.407-3.884), 1.903 (1.134$3.194)$ and 1.598 (0.960-1.125). The patients with DPN had significantly higher prevalence of carotid plaques $(55.8 \%)$ and carotid stenosis $(1.3 \%)$ than non-DPN patients (44.8\%; 0.5\%). However, no statistical association was observed between FT3 quartiles and carotid atherosclerotic lesions.

Conclusions: Lower FT3 within the normal range was independently associated with DPN, but not with carotid atherosclerotic lesions in Chinese type 2 diabetes inpatients with euthyroid function.

\section{Background}

Thyroid dysfunction, whether overt or subclinical, has been widely reported to closely associate with the heart and cardiovascular deteriorations such as coronary artery disease (CAD), atrial fibrillation and heart failure [1-4]. It has also been reported that subclinical hypothyroidism $(\mathrm{SCH})$ is strongly related with diabetic vascular complications, including CAD, diabetic nephropathy (DN) and retinopathy (DR) [5-7]. For example, Chen et al. [7] demonstrated that $\mathrm{SCH}$ in Type 2 diabetics suffered from roughly 3.15-fold risk of DN in the cross-sectional analysis and had 2.93-fold risk for cardiovascular events during the 44.0-months follow-up compared with euthyroid diabetics. However, the studies on the thyroid dysfunction and diabetic peripheral neuropathy (DPN) were extremely scarce.

Recently, it has also been discovered that alterations in thyroid function, even within reference ranges, are connected with atherosclerosis for general population and angina patients [8-10]. The Cardiovascular Health Study and the Rotterdam Study found that euthyroid participants faced the higher risk of heart failure and sudden cardiac death along with the growing free thyroxine (FT4) levels $[10,11]$. However, free triiodothyronine (FT3) as an active metabolite of thyroid hormones was not evaluated in these studies, and the data regarding the relationship of FT3 and atherosclerosis were limited.

Furthermore, several recent studies have reported that close relationships are observed between low-normal FT3 levels and type 2 diabetic macrovascular and microvascular complications [12, 13]. For example, Wu et al. [13] revealed that the occurrence of DN exhibited gradual reduction across the FT3 tertile groups $(59.6 \%, 46.4 \%$, and 
$38.6 \%, \mathrm{p}<0.01$ ) in type 2 diabetes with euthyroid function, and FT3 levels were negatively correlated with urinary albumin-to-creatinine ratio after adjustment for various risk factors according to multiple linear regression analysis.

However, so far there is no data on the association of FT3 with DPN and carotid atherosclerotic lesions in type 2 diabetic patients with normal thyroid function. Therefore, in our study we tried to clarify whether there existed some associations of serum FT3 levels within the normal range with the presence of diabetic peripheral neuropathy and carotid atherosclerotic lesions in Chinese type 2 diabetic patients with euthyroid function.

\section{Materials And Methods}

\section{Subjects and study design}

3231 Chinese patients over the age of 30 hospitalized in the Endocrinology and Metabolism Department of Shanghai Jiao Tong University Affiliated Sixth People's Hospital during the period between June 2005 and May 2012 were collected in our study. And their diagnoses followed the World Health Organization standards in 1999 and American Diabetes Association criteria in $2012[14,15]$. Among these subjects, 754 patients were excluded as follows, 1) lack of thyroid function, neuropathy assessment, carotid ultrasound examination, and complete clinical data; 2 ) type 1 or special type of diabetic patients or patients with acute diabetic complications; 3 ) clinical or diagnosed thyroid disease and goiter, antithyroid drugs or levothyroxine treatments in the present or past, record of thyroidectomy or iodine-131 treatment, history of head and neck external radiotherapy; 4) the levels of thyroid stimulating hormone (TSH), FT3 and FT4 beyond the reference intervals (0.27-4.2 mIU/L for TSH, 3.10$6.80 \mathrm{pmol} / \mathrm{L}$ for FT3, and 12-22 pmol/L for FT4 in this study). Ultimately, 2477 type 2 diabetic patients with euthyroid function were included in our analysis. Written informed consents were got from the whole participants. The study was approved by the Human Research and Ethics Committee of Shanghai Jiao Tong University Affiliated Sixth People's Hospital and adhered to the tenets of the Declaration of Helsinki.

\section{Physical examination and laboratory measurements}

Height, weight, waist circumference, hip circumference and blood pressure of all participants were measured. The body mass index (BMI) was obtained through the weight $(\mathrm{kg})$ divided by the square of height $(\mathrm{m})$. Waist hip ratio was calculated as the waist circumference $(\mathrm{cm})$ divided by the hip circumference $(\mathrm{cm})$. Blood samples were drawn from an antecubital vein for the determination of glycosylated hemoglobin $\mathrm{A} 1 \mathrm{c}(\mathrm{HbA} 1 \mathrm{c})$, fasting plasma glucose (FPG), 2-h postprandial plasma glucose (2 h PPG), fasting C-peptide (FCP), 2-h postprandial C-peptide (2 h PCP), total triglycerides (TTG), total cholesterol (TC), high-density lipoprotein cholesterol (HDL-C), low-density lipoprotein cholesterol (LDL-C), alanine aminotransferase (ALT), creatinine (Cr), serum uric acid (SUA), and Creactive protein (CRP). The 24-h urinary albumin excretion (UAE) was calculated as the mean of the values obtained from three consecutive 24-h urine samples during the period of hospitalization. The homeostasis model assessment for insulin resistance (HOMA-IR) was determined using the HOMA calculator version 2.2.3 [16]. The estimated glomerular filtration rate (eGFR) was calculated using simplified MDRD formula: estimated GFR $($ eGFR $)=186.3 \times($ Serum creatinine $)-1.154 \times($ age $)-0.203(\times 0.742$ if female $)$ [17].

\section{Neuropathy assessment}

Assessment of neuropathy, including neurological symptoms and signs as well as nerve conduction velocity tests, were conducted to the whole subjects. Firstly, following the Toronto Clinical Scoring System [18], the 
licensed physicians evaluated the patients' neurological symptoms and signs, and recorded as positive when the patients had any feelings including pain, numbness, tingling, weakness of foot, reflex abnormalities or ataxia. Secondly, nerve conduction velocity tests were conducted by electromyogram. Briefly, electromyogram was applied to measure the nerve conduction velocities (NCVs) of patients' bilateral median, ulnar, tibia, common peroneal and superficial peroneal nerves while they remained calm and relaxed with their local skin temperature kept constant $\left(>31^{\circ} \mathrm{C}\right)$ by an infrared lamp. And considering Chinese people's NCV reference value, we defined the threshold for lowered NCVs as used [19].

DPN was diagnosed when objects had both obviously clinical DPN features and abnormal test results from nerve conduction velocities (NCVs). And the former was identified when the patients performed at least two positive results among sensory symptoms, signs or abnormal reflections in accordance with symmetrical polyneuropathy at distal end. The latter was recognized when at least one abnormal nerve attribute like amplitude, latency, F-wave or NCV occurred on at least two nerves including median, peroneal and sural nerves.

\section{Carotid Ultrasonography measurements}

Carotid ultrasonography was performed through a machine with a phased-array transducer (Acuson Sequoia 512,

Siemens) and was conducted by the certified and skilled sonographers. The ultrasound scanning protocol used in this study was a modification from procedures used in previous studies [20-22]. That is, the sonographers successively recorded and read the bilateral images of the common carotid arteries $(1 \mathrm{~cm}$ proximal to the dilatation of the carotid bulb), the carotid bulb (identified by the absence of the parallel wall in the common carotid artery), and the internal carotid artery $(1 \mathrm{~cm}$ distal to the tip of the flow divider that separates the external and internal carotid arteries). The intima-media thickness was the distance between the lumen-intima interface and the media-adventitia interface [20]. Carotid intima-media thickness (CIMT) was defined as the mean of the left and right IMTs of the common carotid artery. Carotid plaque was defined as a localized protrusion from the internal part of the vessel wall into the lumen, reaching $50 \%$ of the surrounding IMT value [21, 22]. Carotid stenosis was defined as any degree of narrowing in the carotid arteries caused by carotid plaques [21, 22].

\section{Statistical analyses}

For statistical analysis, SPSS v. 16.0 was used. Figures were created by GraphPad Prism 5.0. Data were expressed as either mean \pm standard deviation or medians (interquartile range $25 \%-75 \%$ ) for continuous variables and percentages (\%) for categorical variables. For continuous variables, One-way ANOVAs with LSDs were used for normal distributions and Kruskal-Wallis $\mathrm{H}$ tests were used for skewed distributions. The chi-squared test was used to compare categorical values. The partial correlations were used to determine the relationships between FT3 and the other clinical variables. Binary logistic regression analysis was performed to evaluate the odds ratio (ORs) of DPN, carotid plaque and stenosis associated with the FT3 quartiles. All p values were two-tailed, and $p<$ 0.05 was considered to be statistically significant.

\section{Results}

\section{Characteristics of the subjects according to FT3 quartiles}

The clinical characteristics of the subjects grouped by FT3 quartiles are presented in Table 1 . The patients were stratified into quartiles by the FT3 levels with the cutoff limits of 3.10-4.10, 4.10-4.40, 4.40-4.72, and 4.72-6.80 
pmol/L. BMI, DBP, FCP, 2 h PCP, TTG, Cr, eGFR, and FT4 progressively increased from the lowest FT3 quartile to the highest quartile, while age, duration of diabetes (DD), 2 h PPG, HbA1c, TC, UAE, and CRP gradually decreased via FT3 quartiles (all $p<0.05$ ) even after adjustment for age and sex. 
Table 1

Clinical characteristics of the subjects

\begin{tabular}{|c|c|c|c|c|c|c|}
\hline Variables & $Q 1(n=612)$ & $Q 2(n=616)$ & $\mathrm{Q} 3(\mathrm{n}=622)$ & $Q 4(n=627)$ & $\begin{array}{l}\mathrm{p} \\
\text { value }\end{array}$ & $\begin{array}{l}{ }^{*} \text { p } \\
\text { value }\end{array}$ \\
\hline FT3 (pmol/L) & $3.10-4.10$ & $4.10-4.40$ & $4.40-4.72$ & $4.72-6.80$ & - & - \\
\hline Age (years) & $64 \pm 11$ & $61 \pm 11$ & $58 \pm 12$ & $55 \pm 11$ & $\dot{0} .001$ & $\begin{array}{l}<.001 \\
0.001\end{array}$ \\
\hline Male (n, \%) & $261(42.6 \%)$ & $298(48.4 \%)$ & $372(59.8 \%)$ & $459(73.2 \%)$ & $\begin{array}{l}<.001 \\
0.00\end{array}$ & $\begin{array}{l}<.001 \\
0.00\end{array}$ \\
\hline *DD (months) & $\begin{array}{l}120(48- \\
180)\end{array}$ & $96(48-156)$ & $90(36-144)$ & $72(24-120)$ & $\dot{0} 001$ & $\dot{0} 001$ \\
\hline Smoking (n, \%) & $114(18.6 \%)$ & $133(21.6 \%)$ & $160(25.7 \%)$ & $241(38.4 \%)$ & $\stackrel{<}{0.001}$ & 0.339 \\
\hline Alcohol $(n, \%)$ & $48(7.8 \%)$ & $71(11.5 \%)$ & $85(13.7 \%)$ & $114(18.2 \%)$ & 0.002 & 0.346 \\
\hline Hypertension (n, \%) & $314(51.3 \%)$ & $330(53.6 \%)$ & $300(48.2 \%)$ & $270(43.1 \%)$ & 0.001 & 0.171 \\
\hline $\mathrm{BMI}\left(\mathrm{kg} / \mathrm{m}^{2}\right)$ & $24.63 \pm 3.68$ & $25.28 \pm 3.38$ & $25.08 \pm 3.45$ & $25.41 \pm 3.38$ & 0.001 & 0.030 \\
\hline WHR & $0.93 \pm 0.35$ & $0.92 \pm 0.07$ & $0.91 \pm 0.06$ & $0.92 \pm 0.06$ & 0.559 & 0.238 \\
\hline $\mathrm{SBP}(\mathrm{mmHg})$ & $133 \pm 18$ & $132 \pm 17$ & $132 \pm 18$ & $131 \pm 16$ & 0.110 & 0.074 \\
\hline $\mathrm{DBP}(\mathrm{mmHg})$ & $79 \pm 10$ & $79 \pm 10$ & $80 \pm 9$ & $82 \pm 9$ & $\stackrel{<}{0.001}$ & 0.007 \\
\hline *FPG (mmol/l) & $\begin{array}{l}7.55(6.04- \\
9.82)\end{array}$ & $\begin{array}{l}7.82(6.31- \\
10.08)\end{array}$ & $\begin{array}{l}7.90(6.26- \\
9.69)\end{array}$ & $\begin{array}{l}7.81(6.40- \\
9.63)\end{array}$ & 0.481 & 0.059 \\
\hline $2 \mathrm{~h}$ PPG (mmol/l) & $14.13 \pm 4.96$ & $14.07 \pm 4.66$ & $13.89 \pm 4.66$ & $13.67 \pm 4.72$ & 0.322 & 0.017 \\
\hline *HbA1C (\%) & $\begin{array}{l}9.3(7.5- \\
11.5)\end{array}$ & $8.7(7.3-10.5)$ & $8.5(7.3-10.2)$ & $8.4(7-9.9)$ & $\stackrel{<}{0.001}$ & $\stackrel{<}{0.001}$ \\
\hline *FCP $(\mathrm{ng} / \mathrm{mL})$ & $\begin{array}{l}1.57(0.89- \\
2.40)\end{array}$ & $\begin{array}{l}1.73(1.10- \\
2.54)\end{array}$ & $\begin{array}{l}1.82(1.20- \\
2.53)\end{array}$ & $\begin{array}{l}1.89(1.30- \\
2.53)\end{array}$ & $\stackrel{<}{0.001}$ & 0.014 \\
\hline 2 h PCP $(\mathrm{ng} / \mathrm{mL})$ & $3.75 \pm 2.87$ & $4.15 \pm 2.74$ & $4.46 \pm 3.01$ & $4.75 \pm 2.71$ & $\begin{array}{l}< \\
0.001\end{array}$ & $\begin{array}{l}< \\
0.001\end{array}$ \\
\hline *HOMA-IR & $\begin{array}{l}4.94(2.98- \\
8.40)\end{array}$ & $\begin{array}{l}4.88(2.96- \\
7.85)\end{array}$ & $\begin{array}{l}4.59(2.91- \\
7.75)\end{array}$ & $\begin{array}{l}4.40(2.72- \\
7.40)\end{array}$ & 0.292 & 0.854 \\
\hline *TTG (mmol/L) & $\begin{array}{l}1.38(0.92- \\
2.08)\end{array}$ & $\begin{array}{l}1.50(1.01- \\
2.18)\end{array}$ & $\begin{array}{l}1.54(1.04- \\
2.41)\end{array}$ & $\begin{array}{l}1.51(1.09- \\
2.10)\end{array}$ & 0.838 & 0.009 \\
\hline $\mathrm{TC}(\mathrm{mmol} / \mathrm{L})$ & $4.88 \pm 1.35$ & $4.84 \pm 1.33$ & $4.79 \pm 1.04$ & $4.67 \pm 0.99$ & 0.009 & 0.009 \\
\hline
\end{tabular}

Values are expressed as the mean \pm S.D, median with interquartile range, or percentages.

*Non-normal distribution of continuous variables.

p-value: The p-values were not adjusted for age and sex for the trend.

${ }^{*} p$-value: The *p-values were adjusted for age and sex for the trend. 


\begin{tabular}{|c|c|c|c|c|c|c|}
\hline Variables & $\mathrm{Q1}(\mathrm{n}=612)$ & Q2(n= 616) & Q3(n = 622) & Q4(n = 627) & $\begin{array}{l}\mathrm{p} \\
\text { value }\end{array}$ & $\begin{array}{l}\text { *p } \\
\text { value }\end{array}$ \\
\hline HDL-C (mmol/L) & $1.17 \pm 0.40$ & $1.14 \pm 0.31$ & $1.10 \pm 0.31$ & $1.10 \pm 0.28$ & $<.001$ & 0.409 \\
\hline LDL-C (mmol/L) & $3.16 \pm 1.03$ & $3.18 \pm 0.98$ & $3.24 \pm 0.93$ & $3.17 \pm 0.92$ & 0.383 & 0.173 \\
\hline *ALT (U/I) & $17(12-26)$ & $19(13-29)$ & $20(14-30)$ & $20(14-30)$ & 0.017 & 0.093 \\
\hline${ }^{*} \mathrm{Cr}(\mu \mathrm{mol} / \mathrm{l})$ & $65(52-80)$ & $65(55-77)$ & $65(55-78)$ & $67(56-78)$ & $\dot{0}_{0.001}$ & $\begin{array}{l}< \\
0.001\end{array}$ \\
\hline *SUA $(\mu \mathrm{mol} / \mathrm{l})$ & $\begin{array}{l}310(251- \\
369)\end{array}$ & $\begin{array}{l}300(248- \\
364)\end{array}$ & $\begin{array}{l}310(265- \\
377)\end{array}$ & $\begin{array}{l}319(267- \\
377)\end{array}$ & 0.023 & 0.911 \\
\hline *UAE (mg/24 h) & $\begin{array}{l}13.05(6.56- \\
40.28)\end{array}$ & $\begin{array}{l}11.73(6.77- \\
32.96)\end{array}$ & $\begin{array}{l}11.25(6.51- \\
27.99)\end{array}$ & $\begin{array}{l}10.14(5.99- \\
24.68)\end{array}$ & 0.024 & 0.002 \\
\hline${ }^{*} \mathrm{eGFR}\left(\mathrm{ml} / \mathrm{min} / 1.73 \mathrm{~m}^{2}\right)$ & $\begin{array}{l}95.35(76.97- \\
118.23)\end{array}$ & $\begin{array}{l}101.37(83.68- \\
117.81)\end{array}$ & $\begin{array}{l}102.78(86.18- \\
120.92)\end{array}$ & $\begin{array}{l}108.25(92.07- \\
126.77)\end{array}$ & $\dot{0}_{0.001}$ & 0.226 \\
\hline${ }^{*} \mathrm{CRP}(\mathrm{mg} / \mathrm{L})$ & $\begin{array}{l}1.46(0.53- \\
3.87)\end{array}$ & $\begin{array}{l}1.09(0.54- \\
2.61)\end{array}$ & $\begin{array}{l}0.96(0.47- \\
2.12)\end{array}$ & $\begin{array}{l}0.93(0.45- \\
1.87)\end{array}$ & $<0.001$ & $<.001$ \\
\hline FT4 (pmol/L) & $15.96 \pm 2.19$ & $16.01 \pm 2.20$ & $16.34 \pm 2.19$ & $16.74 \pm 2.21$ & $<0.001$ & $\begin{array}{l}< \\
0.001\end{array}$ \\
\hline TSH (mIU/L) & $1.74 \pm 0.89$ & $1.74 \pm 0.81$ & $1.69 \pm 0.84$ & $1.67 \pm 0.85$ & 0.405 & 0.655 \\
\hline \multicolumn{7}{|c|}{ Values are expressed as the mean $\pm S . D$, median with interquartile range, or percentages. } \\
\hline \multicolumn{7}{|c|}{ *Non-normal distribution of continuous variables. } \\
\hline \multicolumn{7}{|c|}{$\mathrm{p}$-value: The $\mathrm{p}$-values were not adjusted for age and sex for the trend. } \\
\hline \multicolumn{7}{|c|}{${ }^{*} \mathrm{p}$-value: The ${ }^{*} \mathrm{p}$-values were adjusted for age and sex for the trend. } \\
\hline
\end{tabular}

\section{Comparison of DPN among the FT3 quartile groups}

Figure 1 illustrates the comparison of DPN among the FT3 quartiles in patients. After controlling for age, sex and $\mathrm{DD}$, the prevalence of DPN in patients performed significant decreasing trend across the FT3 quartiles $(23.5 \%$, $20.9 \%, 18.9 \%$, and $11.2 \%$, respectively, $p<0.001$ for the trend; Fig. $1 \mathrm{~A}$ ). Furthermore, the FT3 levels were obviously reduced in the diabetics with DPN compared to those without DPN $(p=0.001$; Fig. 1B). Interestingly, significant sex-related or age-related differences in the prevalence of DPN were found only in the first or fourth FT3 quartiles (Fig. 1C and 1D). However, after further adjusted for smoking, there were neither significant sex-related nor agerelated differences in the prevalence of DPN in any of the quartile groups.

\section{Comparison of the carotid atherosclerotic lesions among the FT3 quartiles}

A comparison of the atherosclerotic lesions among the FT3 quartile groups after adjustments for age, sex and DD is shown in Fig. 2. No statistical associations were successively observed between the FT3 quartiles and CIMT value $(p=0.755)$, the prevalence of carotid plaques $(p=0.052)$ and stenosis $(p=0.090)$ in type 2 diabetes $(F i g .2 A$, $B$ and $C)$. 
Table 2 presents the associations between the FT3 quartiles and the presence of DPN in type 2 diabetes. After controlling for age, sex, smoking, alcohol and hypertension (Model 1), the FT3 quartiles were independently associated with a decreased prevalence of DPN ( $p=0.001$ for trend). After adding other clinical indicators (Model 2 and 3), the FT3 quartiles remained an independent association with a decreased prevalence of DPN $(p=0.001$ and 0.01 for the trends in Model 2, and Model 3). Accordingly, the patients in the lowest to third FT3 quartile had 2.338-, 1.903-, and 1.598-fold risk of DPN, respectively, relative to those in the highest quartile.

Table 2

Association of the FT3 quartiles with DPN in type 2 diabetics

\begin{tabular}{|llllll|}
\hline \multicolumn{1}{|c}{ ORs $(95 \% \mathrm{Cl})$} & \multicolumn{2}{c|}{ P value for trend } \\
\cline { 2 - 5 } & Q4 & Q3 & Q2 & Q1 & \\
\hline Model 1 & 1 (ref) & $1.648(1.101-2.468)$ & $1.867(1.238-2.814)$ & $2.315(1.530-3.503)$ & 0.001 \\
\hline Model 2 & 1 (ref) & $1.746(1.152-2.646)$ & $1.985(1.300-3.031)$ & $2.445(1.589-3.763)$ & 0.001 \\
\hline Model 3 & 1 (ref) & $1.598(0.960-1.125)$ & $1.903(1.134-3.194)$ & $2.338(1.407-3.884)$ & 0.010 \\
\hline Model 1: adjusted for age, sex, smoking, alcohol and hypertension. & \\
\hline Model 2: further adjusted for DD, BMI, WHR, SBP and DBP. & & \\
\hline $\begin{array}{l}\text { Model 3: further adjusted for HbA1c, ALT, eGFR, SUA, UAE, TC, TG, HDL-C, LDL-C, FPG, 2 h PPG, FCP, 2 h PCP, } \\
\text { HOMA-IR, CRP, FT4 and TSH. }\end{array}$ \\
\hline
\end{tabular}

\section{Comparisons of carotid atherosclerotic lesions between the diabetics with and without DPN}

The comparisons of carotid atherosclerotic lesions between the diabetics with and without DPN are illustrated in

Fig. 3. After adjusting for age, sex, DD and smoking, the prevalence of carotid atherosclerotic plaque and stenosis in the diabetics with DPN was significantly higher than that of the diabetics without DPN $(p=0.008$ for plaque, and $p=0.017$ for stenosis) (Fig. 3B and $3 C$ ). However, no significant difference in CIMT were found between the patients with and without DPN (Fig. 3A).

\section{Discussion}

Recent studies have suggested alternations in thyroid hormone levels within the normal range are valuable predictors for adverse cardiac events; So far, almost no any related literature reported the association between FT3 and carotid atherosclerosis. Furthermore, to our knowledge, no studies have investigated the relationship on FT3 and DPN in individuals with normal thyroid function as of now.

In order to fill this gap in the literature, we undertook an analysis to explore whether there existed relationships on FT3, DPN and carotid atherosclerotic lesions in Chinese type 2 diabetic patients with normal thyroid function, and verified that low-normal FT3 concentration was negatively correlated to the incidence of DPN but not to carotid atherosclerotic lesions in type 2 diabetic patients. 
DPN as the most common complication of diabetes, has impacted on around half of the patients which inevitably result in lower living quality and heavier socioeconomic burden [23, 24]. Therefore, it is really necessary to prevent and treat DPN. Although great progress has been made in the study of the pathophysiological mechanisms of DPN, the underlying pathophysiological mechanisms have not been completely elucidated by far. It is generally accepted that not only the hyperglycemia's toxic effects, but also some other elements like dyslipidemia, smoking, DN, and DR, act an important part in the genesis of DPN [25-28]. In line with the above research, we found that in the first FT3 quartile group the prevalence of DPN was higher in males than in females after controlling for age and DD; whereas further adjusted for smoking, no significant sex-related discrepancy were found. Likewise, further controlling for smoking, there were no statistical age-related difference in each FT3 quartile group.

More importantly, we first confirmed that subjects from the first to the third FT3 quartile group had remarkably increased risks of DPN in relation to those in the last quartile group. The literatures on the relationship between thyroid hormone and DPN are quite limited. Recently, Zhao et al. [29] demonstrated that the SCH subjects had higher prevalence and signs of DPN compared with the euthyroid subjects; the multivariate analysis showed that TSH had an independent correlation with DPN and a high TSH level implied to an enhanced risk of DPN in type 2 diabetics. Zhu et al. [30] also showed that serum FT3 levels in normal nerve conduction group were statistically higher than those in abnormal nerve conduction group ( $4.55 \pm 0.65$ vs $4.37 \pm 0.63, p<0.05)$. Consistent with this finding, we also found that the FT3 levels were significantly lower in the diabetic patients with DPN than those without DPN.

DPN is often occurred with DR and DN, but so far only three observations revealed the correlation between FT3 levels and the incidence of DR and DN in type 2 diabetes with normal thyroid function. Resembled with our findings, Wu et al. [13] first discovered that the euthyroid patients with DN got lower FT3 levels compared with those without DN. Zou et al. [31] further found that the prevalence of diabetic kidney disease exhibited a significant downward trend on the FT3 quartiles $(41.1 \%, 30.6 \%, 23.8 \%$, and $18.9 \%, p<0.001)$. Compared with the first FT3 quartile group, the adjusted odds ratio for diabetic kidney disease in the second to fourth FT3 quartile group were 0.655 [95\% confidence interval (Cl): 0.406-1.057], 0.493 (95\% Cl: 0.299-0.813), 0.406 (95\% Cl: 0.2370.697). Likewise, Zou and his team also found the similar results in DR, that is, there was inverse correlation between FT3 within normal range and DR in T2DM patients [32].

So far, the underlying mechanisms on the association between FT3 and DPN remain elusive, but the following enzymes and pathways may be involved in the development of DPN. Firstly, T3 acted directly or indirectly on the endothelial function in vitro by relaxing vascular smooth muscle [33]. The latest study showed that even small fibro neuropathy was related to damaged vascular endothelial function in type 2 diabetic patients [34]; therefore, low FT3 level and DPN may involve to the endothelial dysfunction. Secondly, T3 can facilitate progressive kidney impairment in $\mathrm{db} / \mathrm{db}$ mice through significantly decreasing phosphatidylinositol 3-kinase activity as well as increasing the expression of transforming growth factor- $\beta 1$ [35], which were also reported to accelerate the progression of DPN [36, 37]. Finally, in vivo and in vitro experimental models further showed that 3, 5Diiodothyronine, a natural metabolite of T3, could ameliorate DN by regulating Sirtuin 1 [38], which also played a vital role in prevention and reversal of DPN [39].

On the contrary, we found that FT3 didn't have remarkable association with atherosclerotic lesions in type 2 diabetic patients. Currently no paper regarding the relationship of FT3 and carotid atherosclerosis are obtainable, several studies have reported there exists a powerful connection on FT3 and the presence and severity of CAD 
which had been well-studied in euthyroid individuals [40,41]. For example, Ertaş et al. [40] found that FT3 levels within the normal range were negatively related to the presence and severity of CAD for patients undergoing coronary angiography. Daswani et al. [41] also found that the genesis of CAD was in connection with lower serum FT3 levels; the concentrations of serum FT3 were associated with the Gensini score as well which could make an independent prediction on the severity of CAD in euthyroid stable angina patients.

Lately, some scholars further explored the associations of FT3 Levels and macrovascular complications in type 2 diabetic patients with normal thyroid function [42, 43]. Different from us, Wang et al. [42] showed that diabetic patients with low-normal FT3 level were more likely to suffer from macrovascular complications than those with mid-and-high normal FT3 level. Hu et al. [43] also revealed that there was a remarkable relationship between diabetic macrovascular complications and normal FT3 (OR $=0.534,95 \% \mathrm{Cl} 0.358-0.796)$. This discrepancy between above studies and our studies can be explained by the fact that the definition of macrovascular complications atherosclerosis included atherosclerosis of the aorta, coronary, basilar, carotid in above studies, while our current study mainly focused on carotid atherosclerosis. Thus, our study confirmed for the first time that FT3 within the normal range didn't have an independent association with carotid atherosclerosis, which was considered as an early biomarker of cardiovascular disease, in type 2 diabetic patients with euthyroid function.

Additionally, we found that the incidence of carotid atherosclerotic plaque and stenosis in type 2 diabetic patients with DPN was significantly higher than those without DPN; whereas no significant difference in CIMT was observed between them, which suggested that DPN may be related to late rather than early carotid atherosclerotic lesions in type 2 diabetes. This finding further provided strong evidence that diabetes-induced endothelial dysfunction was an important and initial factor in the development of diabetic vascular complications [44, 45].

Some limitations in our study need to be mentioned. Firstly, further studies need to verify whether our results can be applied to ethnicity or other types of diabetes as the participants in our report were Chinese Han type 2 diabetic patients. Secondly, the causal connection on the reduced FT3 and DPN need further verification due to the nature of cross-sectional study. Thus, prospective research should be made to explore the connection on low-grade FT3 and the occurrence of DPN in various population and other type of diabetes. Thirdly, other medications for the patients were not considered in our study expect the thyroid-related drugs.

\section{Conclusions}

Our results found that the decreased FT3 was independently associated with the presence of DPN but not with carotid atherosclerosis in type 2 diabetes with euthyroid function. It hinted the concentration of FT3 less than $4.10 \mathrm{pmol} / \mathrm{L}$ increased nearly 2.5 -fold risk of DPN in comparison with FT3 above $4.72 \mathrm{pmol} / \mathrm{L}$. FT3, an efficient and inexpensive indicator, may be a simple and helpful serum marker and a potential pathogenic factor of DPN. Further studies are needed to determine underlying associations and mechanisms between FT3 and DPN in order to provide a potential strategy for the prevention and treatment of DPN in type 2 diabetes.

\section{Abbreviations}

CAD: coronary artery disease; SCH: subclinical hypothyroidism; DN: diabetic nephropathy; DR: diabetic retinopathy; DPN: diabetic peripheral neuropathy (DPN); TSH: thyroid stimulating hormone; FT4: free thyroxine; FT3: free triiodothyronine; BMI: body mass index; WHR: waist-to-hip ratio; SBP: systolic blood pressure; DBP: diastolic blood pressure; HbA1c: glycated hemoglobin A1c; FPG: fasting plasma glucose; 2-h PPG: 2-h 
postprandial plasma glucose; FCP: fasting C-peptide; 2 h PCP: 2-h postprandial C-peptide; HOMA-IR: homeostasis model assessment for insulin resistance; TTG: total triglycerides; TC: total cholesterol; HDL-C: high-density lipoprotein cholesterol; LDL-C: Iow-density lipoprotein cholesterol; ALT: alanine aminotransferase; Cr: creatinine; SUA: serum uric acid; CRP: C-reactive protein; UAE: 24-h urinary albumin excretion; eGFR: glomerular filtration rate; NCV: nerve conduction velocity; CIMT: carotid intima-media thickness.

\section{Declarations}

\section{Acknowledgements}

Not applicable.

\section{Authors' Contributions}

JBL designed the study, supervised the work, reviewed and edited the article. SL, JWW, and ZHZ collected samples and clinical data. MFL and JFK researched the data, performed the statistical analysis and wrote the manuscript. All authors have read and approved the final manuscript.

\section{Funding}

This work was supported by the National Natural Science Foundation of China (grant number 81502316), the Translational Medicine National Key Science and Technology Infrastructure Open Project (grant number TMSK2021-116), and the Exploratory Clinical Research Project of Shanghai Jiao Tong University Affiliated Sixth People's Hospital (grant number ynts202105).

\section{Availability of data and materials}

The datasets used and/or analysed during the current study are available from the corresponding author on reasonable request.

\section{Ethics approval and consent to participate}

The study was approved by the human research ethic committee of Shanghai Jiao Tong University Afliated Sixth People's Hospital, and all patients had signed written informed consent.

\section{Consent for publication}

Not applicable.

\section{Competing interests}

The authors declare that they have no competing interests.

\section{References}

1. Klein I. Thyroid and the heart: the intimacy is strained. Thyroid. 2011;21(5):469-70.

2. Pearce EN, Yang Q, Benjamin EJ, et al. Thyroid function and left ventricular structure and function in the Framingham Heart Study. Thyroid. 2010;20(4):369-73. 
3. Jabbar A, Pingitore A, Pearce SH, et al. Thyroid hormones and cardiovascular disease. Nat Rev Cardiol. 2017;14(1):39-55.

4. Asvold BO, Bjøro T, Platou C, et al. Thyroid function and the risk of coronary heart disease: 12-year follow-up of the HUNT study in Norway. Clin Endocrinol (Oxf). 2012;77(6):911-7.

5. Jia F, Tian J, Deng F, et al. Subclinical hypothyroidism and the associations with macrovascular complications and chronic kidney disease in patients with Type 2 diabetes. Diabet Med. 2015;32(8):1097103.

6. Yang JK, Liu W, Shi J, et al. An association between subclinical hypothyroidism and sight-threatening diabetic retinopathy in type 2 diabetic patients. Diabetes Care. 2010;33(5):1018-20.

7. Chen RL, Lin HD. Subclinical hypothyroidism is a risk factor for nephropathy and cardiovascular diseases in Type 2 diabetic patients. Diabet Med. 2007;24(12):1336-44.

8. Auer J, Berent R, Weber T, et al. Thyroid function is associated with presence and severity of coronary atherosclerosis. Clin Cardiol. 2003;26(12):569-73.

9. Yun $\mathrm{KH}$, Jeong $\mathrm{MH}$, Oh SK, et al. Relationship of thyroid stimulating hormone with coronary atherosclerosis in angina patients. Int J Cardiol. 2007;122(1):56-60.

10. Cappola AR, Arnold AM, Wulczyn K, Carlson M, Robbins J, Psaty BM. Thyroid function in the euthyroid range and adverse outcomes in older adults. J Clin Endocrinol Metab 2015;100(3):1088-96.

11. Chaker L, van den Berg ME, Niemeijer MN, et al. Thyroid Function and Sudden Cardiac Death: A Prospective Population-Based Cohort Study. Circulation. 2016;134(10):713- 22.

12. Hu Y, Yan Z, Pan C. Associations of Thyroid Hormone Levels and Macrovascular Complications in Euthyroid Type 2 Diabetic Patients. Diabetes Metab Syndr Obes. 2021;14:2683-2691.

13. Wu J, Li X, Tao Y, et al. Free Triiodothyronine Levels Are Associated with Diabetic Nephropathy in Euthyroid Patients with Type 2 Diabetes. Int J Endocrinol. 2015;204893.

14. Alberti KG, Zimmet PZ. Definition, diagnosis and classification of diabetes mellitus and its complications. Part 1: diagnosis and classification of diabetes mellitus provisional report of a WHO consultation. Diabet Med. 1998;15(7):539-53.

15. American DA. Standards of medical care in diabetes-2012. Diabetes Care. 2012; 35(Suppl 1):S11-63.

16. Levy JC, Matthews DR, Hermans MP. Correct homeostasis model assessment (HOMA) evaluation uses the computer program. Diabetes Care. 1998;21(12):2191-2.

17. National KF. K/DOQI clinical practice guidelines for chronic kidney disease: evaluation, classification, and stratification. Am J Kidney Dis. 2002;39(2 Suppl 1):S1-266.

18. Bril V, Perkins BA. Validation of the Toronto Clinical Scoring System for diabetic polyneuropathy. Diabetes Care. 2002;25(11):2048-52.

19. He R, Hu Y, Zeng H, et al. Vitamin D deficiency increases the risk of peripheral neuropathy in Chinese patients with type 2 diabetes. Diabetes Metab Res Rev. 2017;33(2).

20. Epidemiology of Diabetes Interventions and Complications (EDIC) Research Group. Effect of intensive diabetes treatment on carotid artery wall thickness in the epidemiology of diabetes interventions and complications. Epidemiology of Diabetes Interventions and Complications (EDIC) Research Group. Diabetes. 1999 Feb;48(2):383-90. 
21. Li MF, Ren Y, Zhao CC, et al. Prevalence and clinical characteristics of lower limb atherosclerotic lesions in newly diagnosed patients with ketosis-onset diabetes: a cross-sectional study. Diabetol Metab Syndr. 2014;6:71.

22. Li MF, Zhao CC, Li TT, et al. The coexistence of carotid and lower extremity atherosclerosis further increases cardio-cerebrovascular risk in type 2 diabetes. Cardiovasc Diabetol. 2016;15:43.

23. Argoff CE, Cole BE, Fishbain DA, et al. Diabetic peripheral neuropathic pain: clinical and quality-of-life issues. Mayo Clin Proc. 2006;81(4 Suppl):S3-11.

24. AlSufyani MH, Alzahrani AM, Allah AA, et al. Prevalence of painful diabetic peripheral neuropathy and its impact on quality of life among diabetic patients in Western region, Saudi Arabia. J Family Med Prim Care. 2020;9(9):4897-4903.

25. Grisold A, Callaghan BC, Feldman EL. Mediators of diabetic neuropathy: is hyperglycemia the only culprit? Curr Opin Endocrinol Diabetes Obes. 2017;24(2):103- 111.

26. Valensi P, Giroux C, Seeboth-Ghalayini B, et al. Diabetic peripheral neuropathy: effects of age, duration of diabetes, glycemic control, and vascular factors. J Diabetes Complications. 1997;11(1):27-34.

27. Tesfaye S, Chaturvedi N, Eaton SE, et al. Vascular risk factors and diabetic neuropathy. N Engl J Med. 2005;352(4):341-50.

28. Dyck PJ, Kratz KM, Karnes JL, et al. The prevalence by staged severity of various types of diabetic neuropathy, retinopathy, and nephropathy in a population-based cohort: the Rochester Diabetic Neuropathy Study. Neurology. 1993; 43(4):817-24.

29. Zhao W, Zeng $H$, Zhang $X$, et al. A high thyroid stimulating hormone level is associated with diabetic peripheral neuropathy in type 2 diabetes patients. Diabetes Res Clin Pract. 2016;115:122-9.

30. Zhu FF, Yang LZ. The Association Between the Levels of Thyroid Hormones and Peripheral Nerve Conduction in Patients with Type 2 Diabetes Mellitus. Exp Clin Endocrinol Diabetes. 2018;126(8):493-504.

31. Zou J, Tian F, Zhang Y, et al. Association between Thyroid Hormone Levels and Diabetic Kidney Disease in Euthyroid Patients with Type 2 Diabetes. Sci Rep. 2018;8(1):4728.

32. Zou J, Li Z, Tian F, et al. Association between Normal Thyroid Hormones and Diabetic Retinopathy in Patients with Type 2 Diabetes. Biomed Res Int. 2020;2020:8161797.

33. Ojamaa K, Klemperer JD, Klein I. Acute effects of thyroid hormone on vascular smooth muscle. Thyroid. 1996;6(5):505-12.

34. Ando A, Miyamoto M, Saito N, et al. Small Fibre Neuropathy Is Associated With Impaired Vascular Endothelial Function in Patients With Type 2 Diabetes. Front Endocrinol (Lausanne). 2021;12:653277.

35. Lin Y, Sun Z. Thyroid hormone ameliorates diabetic nephropathy in a mouse model of type II diabetes. J Endocrinol. 2011;209(2):185-91.

36. Li R, Li Y, Wu Y, et al. Heparin-Poloxamer Thermosensitive Hydrogel Loaded with bFGF and NGF Enhances Peripheral Nerve Regeneration in Diabetic Rats. Biomaterials. 2018;168:24-37.

37. Hussain G, Rizvi SA, Singhal S, et al. Serum levels of TGF- $\beta 1$ in patients of diabetic peripheral neuropathy and its correlation with nerve conduction velocity in type 2 diabetes mellitus. Diabetes Metab Syndr. 2016;10(1 Suppl 1):S135-9.

38. Shang G, Gao P, Zhao Z, et al. 3,5-Diiodo-I-thyronine ameliorates diabetic nephropathy in streptozotocininduced diabetic rats. Biochim Biophys Acta. 2013;1832(5):674-84. 
39. Chandrasekaran K, Salimian M, Konduru SR, et al. Overexpression of Sirtuin 1 protein in neurons prevents and reverses experimental diabetic neuropathy. Brain. 2019;142(12):3737-3752.

40. Ertaş F, Kaya H, Soydinç MS. Low serum free triiodothyronine levels are associated with the presence and severity of coronary artery disease in the euthyroid patients: an observational study. Anadolu Kardiyol Derg. 2012;12(7):591-6.

41. Daswani R, Jayaprakash B, Shetty R, et al. Association of Thyroid Function with Severity of Coronary Artery Disease in Euthyroid Patients. J Clin Diagn Res. 2015;9(6):0C10-3.

42. Wang L, Chen T, Yu J, et al. Clinical Associations of Thyroid Hormone Levels with the Risk of Atherosclerosis in Euthyroid Type 2 Diabetic Patients in Central China. Int J Endocrinol. 2020;2020:2172781.

43. Hu Y, Yan Z, Pan C. Associations of Thyroid Hormone Levels and Macrovascular Complications in Euthyroid Type 2 Diabetic Patients. Diabetes Metab Syndr Obes. 2021;14:2683-2691.

44. Shi Y, Vanhoutte PM. Macro- and microvascular endothelial dysfunction in diabetes. J Diabetes. 2017;9(5):434-449.

45. Roustit M, Loader J, Deusenbery C, et al. Endothelial Dysfunction as a Link Between Cardiovascular Risk Factors and Peripheral Neuropathy in Diabetes. J Clin Endocrinol Metab. 2016;101(9):3401-8.

\section{Figures}

A

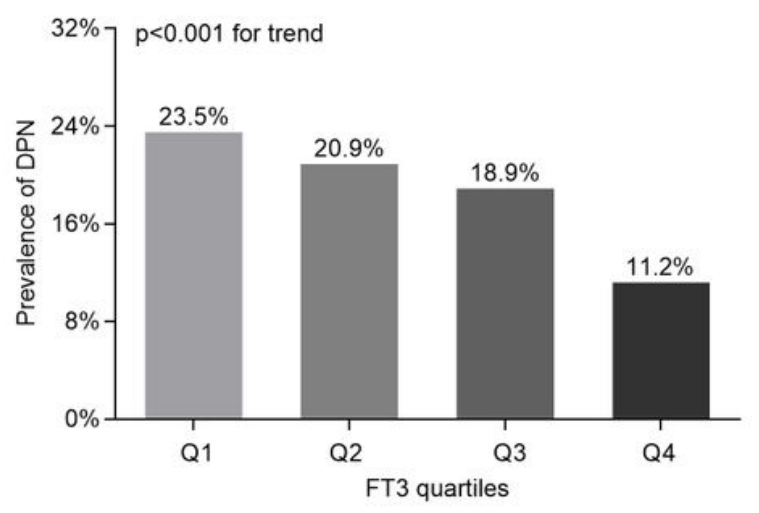

C

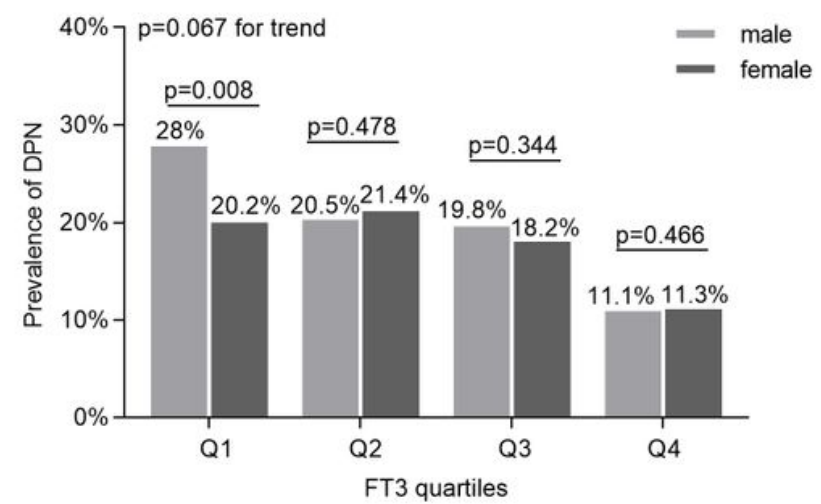

B

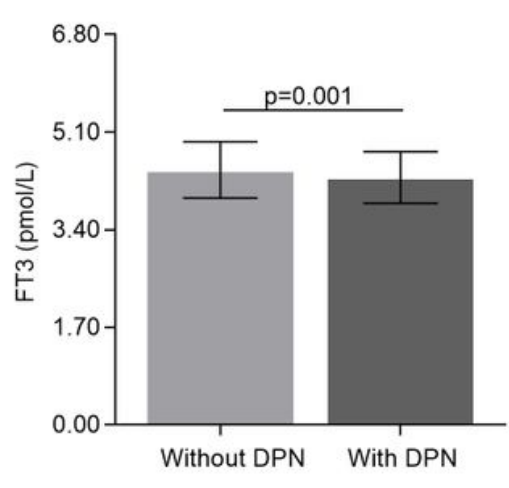

D

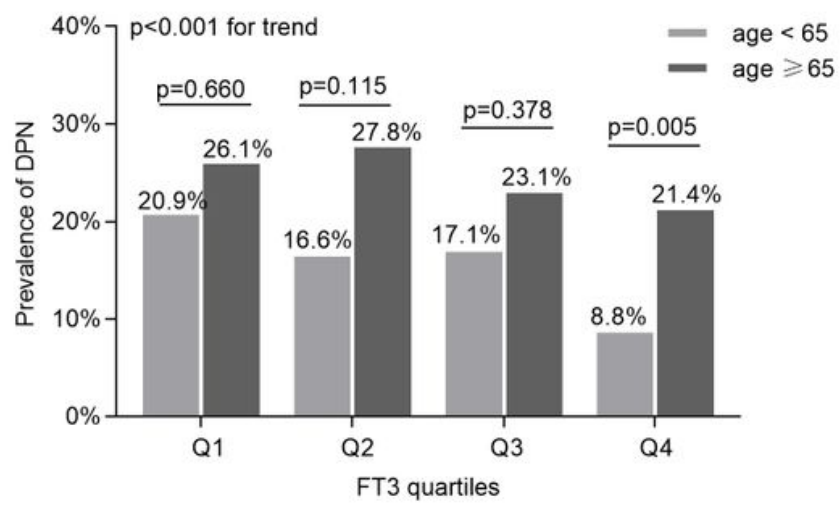

Figure 1 
Comparison of DPN among the FT3 quartile groups. (A) Comparison of the prevalence of DPN among the four groups after adjusting for age, sex, and DD. The $p$-value for the four-group comparison was $<0.001$. (B) Comparison of the FT3 levels between the diabetics with and without DPN after adjusting for age, sex, and DD. The p-value was 0.001. (C) Comparison of the prevalence of DPN stratified by sex in each FT3 quartile group after adjusting for age and DD. The $p$-value for the four-group trend was 0.067. (D) Comparison of the prevalence of DPN stratified by age in each FT3 quartile group after adjusting for sex and DD. The p-value for the four-group trend was $<0.001$.
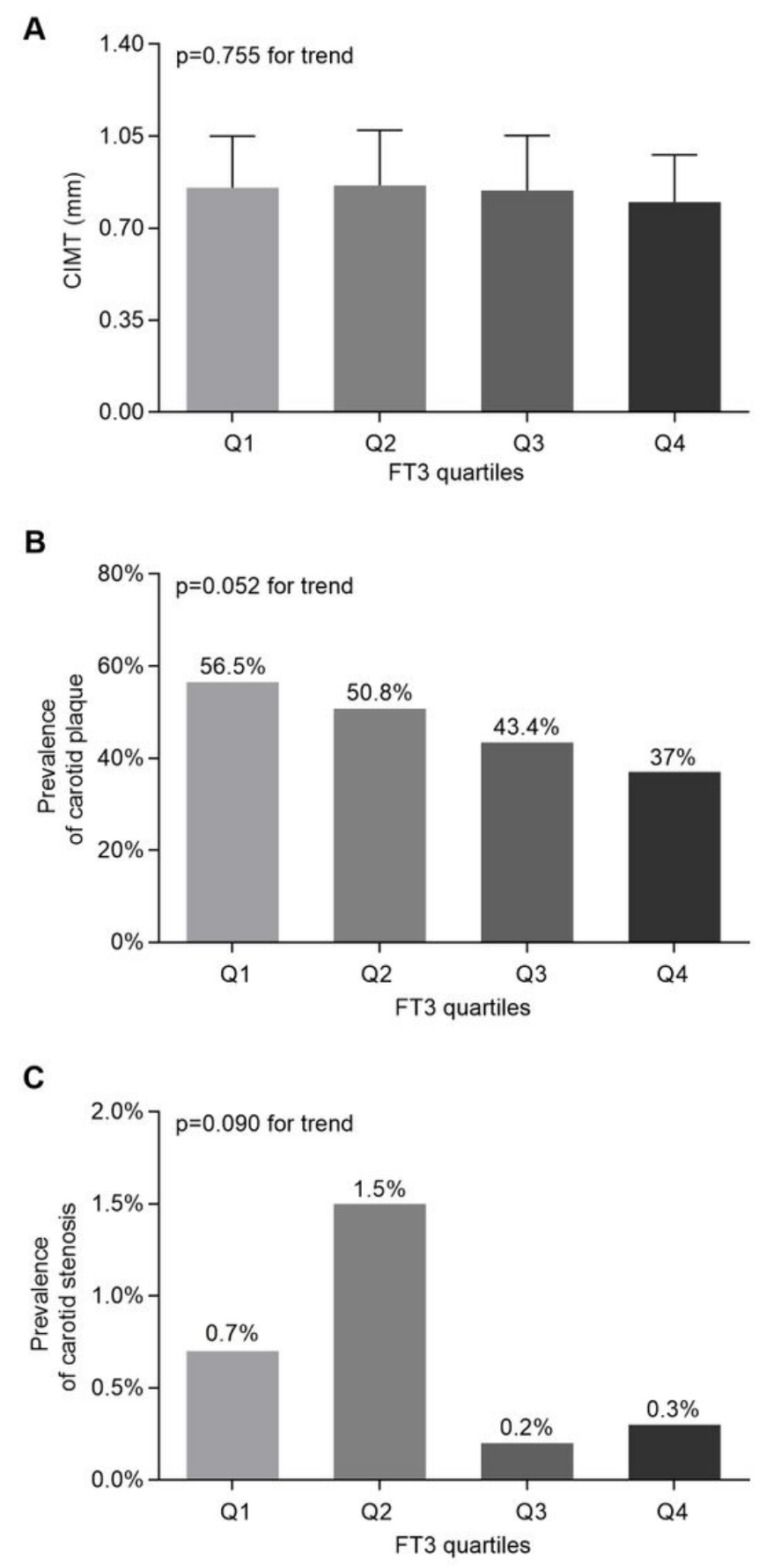

Figure 2 
Comparison of carotid atherosclerotic lesions among FT3 quartile groups after adjusting for age, sex, and DD. (A) Comparison of mean CIMT among the four groups. The p-value for the four-group comparison was 0.755 . (B) Comparisons of the prevalence of carotid plaque among four groups. The $p$-value for four group comparison was 0.052. (C) Comparison of the prevalence of carotid stenosis among the four groups. The p-value for the fourgroup comparison was 0.090 .
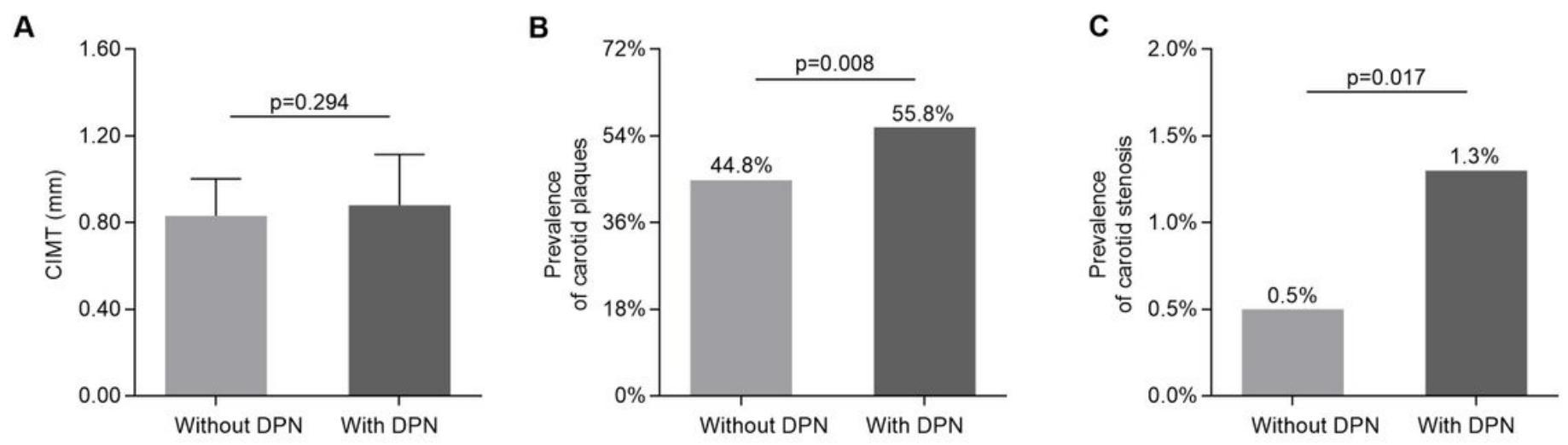

\section{Figure 3}

Comparison of carotid atherosclerotic lesions between the diabetics with and without DPN after adjusting for age, sex, DD, smoking, and alcohol. (A) Comparison of the mean CIMT value between the diabetics with and without DPN. (B) Comparison of the prevalence of carotid plaque between the diabetics with and without DPN. (C) Comparison of the prevalence of carotid stenosis between the diabetics with and without DPN. 